\title{
Preoperative Risk-Stratification of Pediatric Malignant Brainstem Glioma based on a validated nomogram: A SEER based Analysis
}

wenqiang Che

Jinan University First Affiliated Hospital

Jun Lyu

Jinan University First Affiliated Hospital

Chengzhuo Li

Jinan University First Affiliated Hospital

Xiangyu Wang ( $\square$ wang_xy123@126.com )

Jinan University First Affiliated Hospital https://orcid.org/0000-0001-5103-433X

\section{Research Article}

Keywords: nomogram, SEER, brainstem malignant glioma, cancer-specific survival, pediatric

Posted Date: May 24th, 2021

DOI: https://doi.org/10.21203/rs.3.rs-368683/v2

License: (9) This work is licensed under a Creative Commons Attribution 4.0 International License. Read Full License 


\section{Abstract}

Purpose: Pediatric patients diagnosed with brainstem malignant gliomas (BSMGs) have a poor prognosis. Our study aimed to construct and validate a prognostic nomogram to predict the cancerspecific survival preoperatively (CSS) rates and then a risk-stratification given to these patients.

Methods: From 1998 to 2016, we extracted patients' data from Surveillance Epidemiology and End Results (SEER) database. A total of 1160 patients were enrolled and randomly divided into training and validating groups. Subsequently, the Cox regression analysis was used to screen variables. Then, the nomogram was constructed. Lastly, we calculated C-indexes and plotted calibration curves and the utility of decision curve analyses (DCAs) to assess our survival model's benefits.

Result: Here, after multivariate cox regression analysis, we established four variables for constructing nomogram for CSS rates. Subsequently, the C-index, the area under the receiver operating characteristic curve, and calibration curves were used to confirm the nomogram's good performance. DCAs of the nomogram indicated that both groups obtained good 1-, 3-, and 5-year net benefits.

Conclusion: The nomogram model for preoperatively predicting CSS provided a convenient and practical tool to assess pediatric patients' prognosis with BSMG.

\section{Introduction}

Among solid tumors, brain tumors are one of the leading causes of mortality in children globally.(Ostrom and Gittleman et al., 2018) Studies have shown that about $10-20 \%$ of childhood central nervous system tumors are located in the brainstem(Albright and Price et al., 1983) and are associated with a dismal prognosis.(Fisher and Breiter et al., 2000) Recently, the Central Brain Tumor Registry of the United States (CBTRUS) reported that the brainstem glioma accounts for $4.2 \%$ of all gliomas.(Dolecek and Propp et al., 2012) However, the fact that brainstem glioma is insensitive to chemotherapy and radiotherapy makes non-surgical therapy invalid.(Freeman and Farmer, 1998; Hargrave and Bartels et al., 2006) Besides, their complex anatomical structure makes surgical treatment more difficult.(Sun and Xu et al., 2020) Therefore, optimal therapy for brainstem malignant glioma (BSMG) is still under investigation.

The United States National Cancer Institute's Surveillance, Epidemiology, and End Results (SEER) is a nationally representative population-based publicly available cancer database. The SEER contains more than 8 million cancer cases from 1973 to 2016, almost 28\% of the United States population.(Fuchs and Kreil et al., 2002) This database records the demographic information, tumor location, pathological information, morphology, stage of diagnosis, therapy, and prognosis of millions of malignant tumors or carcinoma patients. Besides, it provides useful data for clinical research of cancer. For instance, medical nomograms use biological and clinical variables, such as tumor grade, patient age, and sex, to determine the statistical prognostic models that generate the probability of clinical events, like cancer recurrence or death, for a particular individual.(Balachandran and Gonen et al., 2015) In this study, we extracted pediatric patients' data with primary BSMG cases from the SEER database and then constructed 
nomogram to preoperatively predict patient survival rate, guide clinical prognosis and treatment decisions.

\section{Methods}

We extracted patients' data from the SEER database (1975-2016), which was released in April 2019, based on the November 2018 submission using the SEER ${ }^{\star}$ Stat software version 8.3.8. The inclusion criteria for all enrolled cases included: primary-site codes; C71.7, brain stem; and morphology codes such as 9380/3 (Glioma, malignant), 9401/3 (Astrocytoma, anaplastic), 9440/3 (Glioblastoma, NOS), and $9451 / 3$ (Oligodendroglioma, anaplastic). This study focused on patients who were diagnosed between 1998 and 2016 and were younger than 18 years. On the other hand, the following exclusion criteria were used: 1 . Survival months were unknown; 2 . Not the first tumor.

Subsequently, we extracted the following variables: age, sex, race, age/years at diagnosis, histologic type, survival months, and cause-specific mortality. Consequently, we grouped all variables into different subgroups to make data suitable for analysis using the Rstudio software and to optimize the model. These subgroups included: sex (male, female), race (white, black, and other), age at diagnosis $(<=9,>9)$, tumor size ( $<=25 \mathrm{~mm},>25 \mathrm{~mm}$, and Unkown), and histologic type (low-grade, high-grade, and mixed), except survival months (defined as a continuous variable). We used the X-tile software to define the groups of age/years at diagnosis and tumor size. Our primary outcomes were cancer-specific survival (CSS), and all patients were randomly divided into training and validation groups at a ratio of 7:3. Then, a log-rank test was applied to assess the survival difference between these two groups. Afterward, we did a multivariable Cox and backward stepwise Cox regression analysis to pick out prognostic variables in the training group to fit the model. Thereafter, the $\mathrm{C}$-index was calculated in both the training and validation groups to assess internal validation. Later on, the following packages such as; "rms", "foreign", and "survival" in RStudio software were used in building nomogram for predicting survival rates of CSS in pediatric patients with BSMGs and were over 1-, 3-, and 5-year.

Our study also applied a calibration method with bootstrapping values to demonstrate the correlation between the actual and the predicted probability, and the operation characteristic curve (ROC) of both the training and the validation groups were plotted. Lastly, the area under the receiver operating characteristic curve (AUC) was used to assess the nomogram's prediction accuracy, whereas the decision curve analyses (DCAs) were plotted to test the clinical value of the predictive models. Notably, all of the statistical analyses were performed using the RStudio software version 1.3.1093 (http://www.rstudio.com)

Our study protocol was approved by the Ethical Committee of the first hospital affiliated to Jinan University, and all data deposited in the SEER database are freely available to all users without any restrictions.

\section{Results}




\section{Patient Baseline Characteristics}

Table 1 illustrates the baseline demographic characteristics of the two groups. From 1998 to 2016, a total of 1160 patients matched our inclusion criteria as outlined above. We used the X-tile software to calculate the following optimal cutoffs; for the age at diagnosis (Figure 7A), tumor size (Figure 7B), and years at diagnosis (Figure 7C). Of all patients, 819 (70.60\%) patients were younger than nine years, whereas the remaining 341 (29.40\%) were older than nine. Among these patients, 562 (48.45\%) were male, and a total of 310 patients $(26.72 \%)$ were diagnosed before the year 2003 . Moreover, white was the predominant race. The tumor size in $166(14.31 \%)$ patients was smaller than $25 \mathrm{~mm}$, whereas that of 449 (38.71\%) patients was unknown. In 19 (1.64\%) patients, the tumors were located in the left, and 18 $(1.55 \%)$ in the right.

\section{Multivariate cox regression results}

At a ratio of 7:3, patients were randomly divided into training and validating groups, respectively. Next, we used the log-rank test to explain no significant survival difference between the training and validation groups ( $p=0.9$ ). Subsequently, significant variables in the results of the stepwise-backward Cox regression model and multivariate-Cox regression analyses of CSS were entered into the construction of a nomogram. As illustrated in Figure 1 and Figure 2 (A-D), significant variables contained age at diagnosis, years at diagnosis, tumor size, and histological grade.

\section{Nomogram construction and validation}

As illustrated in Figure 2, the constructed nomogram showed that tumor size was the most vital factor influencing prognosis and was followed by the histological grade, age at diagnosis, and years at diagnosis, respectively. Subsequently, we applied the C-index to assess the performance of the nomogram. Here, the CSS prediction results showed that the $\mathrm{C}$-index value in the training group was 0.649 , whereas that of the validating group was 0.652 . Among the training groups, $1-, 3-$, and 5 -year relative survival AUCs were $0.695,0.803$, and 0.803 (Figure $3 A$ ). On the other hand, as illustrated in Figure $3 B$, the validation group AUC values for the different years were $0.680,0.835$, and 0.843 , respectively. As demonstrated in Figure 4, the calibration curves show excellent consistency between nomogram in CSS predictions and actual observations in 1-, 3-, and 5-year survival rates. Moreover, as shown in Figure 5, the DCA curves of 1-, 3-, and 5-year show that the CSS model could generate net benefits in the training and validation groups. Additionally, we conducted a risk group stratification of the total risk score calculated using the nomogram. Pediatric patients with BSMG were divided into two categories: low risk (total points $<=92.3$ ) and high risk (total points $>92.3$ ). A Kaplan-Meier product-limit estimate was applied to estimate the survival curve between two risk categories (Figure $6 \mathrm{E}$ ).

\section{Discussion}

Previously, studies have shown that malignant brainstem gliomas in pediatric patients have a poor prognosis.(Maxwell and Luksik et al., 2018; Khalid and Kelly et al., 2019) Therefore, there is a need to 
discover a new complete prognostic scoring system for an accurate and useful prognosis assessment. A nomogram is a graphical representation of complex mathematical formulas.(Grimes, 2008) The nomogram showed much more convenience and accuracy when used in clinical applications. A higher Cindex means a better predictive value.(Wang and Li et al., 2013; Zhu and Zhu et al., 2016) The nomogram utility has been assessed in many fields, like bladder cancer,(Yang and Bai et al., 2020) esthesioneuroblastomas,(Jiang and Lin et al., 2020) non-metastatic breast cancer,(Qian and Jia et al., 2020) or in neurosurgery of cancers like glioblastoma(Li and He et al., 2020) and so forth. Of note, this is the first research to introduce nomogram into pediatric primary BSMG before the onset of therapies.

It is commonly believed that the prognosis of cancer patients depreciates with age. Previous studies of adult patients with brainstem gliomas have shown that younger age is a prognostic factor for improved survival.(Guillamo and Monjour et al., 2001; Theeler and Ellezam et al., 2015; Esteve-Codina and Alameda et al., 2020) Doyle et al. reported that those patients whose age was more than 50 years significantly signified a worse prognosis than younger cohorts.(Doyle and Khalafallah et al., 2019) However, in pediatric patients, the situation was different. Our study showed that the patients older than nine years had a better prognosis. In our opinion, this could be due to the patient's younger age, which decreased their tolerance to the disease and treatment measures. Notably, the research by Sun et al. supported our findings. Here, a significantly better prognosis was observed in patients whose age was more than ten years.(Sun and Xu et al., 2020)

Regarding the size of the tumor, the large size (defined as tumors with dimensions greater than $25 \mathrm{~mm}$ ) was an independent predictor of mortality in our patient cohort. Maxwell et al. established that the tumor size, which was larger than $34 \mathrm{~mm}$ was a significantly worse predictor of mortality in his cohort.(Maxwell and Luksik et al., 2018) Large size ( $>2 \mathrm{~cm}$ ), enhancement, and invasion of adjacent brainstem structures on head MRI are signs of more aggressive tectal lesions(Rengachary and Rauf, 2012). Besides, the brainstem contained an extremely high density of critical structures.(Scott, 2004; Mukherjee and Antar et al., 2020) Nevertheless, as the tumor diameter increased, these critical structures are more likely to be compressed, which partly defined the surgiCal complexity.

Interestingly, pediatric BSMGs diagnosed after 2003 had a significantly better prognosis than that before 2003. It has been gradually recognized that brainstem gliomas comprise a heterogeneous group of tumors, and the patients could benefit from introducing the concept of precision and individualized therapeutic methods.(Wong and Brown et al., 2019) As the study progressed, a better understanding of the biology of BSMGs would improve treatment options and outcomes undoubtedly. (Reyes-Botero and Mokhtari et al., 2012) In previous studies, the biopsy is warranted in cases only when tumor progression. (Stark and Fritsch et al., 2005) Nevertheless, subsequent research suggested that brainstem stereotactic biopsy in children was a safe procedure and could alter the treatment recommendations.(Pincus and Richter et al., 2006) There is minimal long-term benefit from chemotherapy, and the mainstay of treatment for BSMGs is radiotherapy. After 2003, it has been demonstrated that the use of radiochemotherapy may improve treatment efficacy.(Marcus and Dutton et al., 2003; Sanghavi and 
Needle et al., 2003; Bernier-Chastagner and Grill et al., 2005; Hargrave and Bartels et al., 2006) All of the abovementioned facts can directly lead to better survival among pediatrics diagnosed after 2003.

According to the 2016 World Health Organization (WHO) of the Central Nervous System Grading Standard, gliomas are divided into four grades (I-IV), depending on the histological and molecular features.(Louis and Perry et al., 2016) High-grade gliomas (WHO grade III-IV) are more aggressive and invasive than low-grade (WHO grade I-II), and high-grade brainstem gliomas have shown poorer survival rates in pediatrics(Jallo and Biser-Rohrbaugh et al., 2004; Sun and Wan et al., 2013), which is in line with our findings. Lesions with focal enhancement on head MRI in pediatric BSMGs were found mostly lowgrade glioma.(Lesniak and Klem et al., 2003) Necrosis and inhomogeneous contrast enhancement were regarded as significant predictors of high-grade brainstem gliomas.(Moharamzad and Sanei Taheri et al., 2018) Recurrent mutations of a regulatory histone-H3F3A,(Schwartzentruber and Korshunov et al., 2012) gain of H3K27 methylation at p16INKA4A,(Chan and Fang et al., 2013) and upregulation of MYCN in G34R/v mutated(Bjerke and Mackay et al., 2013) were associated with carcinogenesis, tumor progression, and the risk of pediatric high-grade gliomas and had a significant effect on survival.

We divided pediatrics patients into low and high-risk groups according to their total points calculated from the nomogram. Kaplan Meier survival analysis showed significant differences in cancer-specific survival between low and high-risk groups. Thus, particular attention was given to these pediatric BSMGs with total points higher than 92.3 or tumor diameters larger than $2 \mathrm{~cm}$ because of their poor outcomes.

In this study, we performed calibration and validation after the nomogram was constructed. Here, the receiver operating characteristics (ROC) were plotted using the calibration method, and the respective areas under the receiver operating characteristic curve (AUC) were calculated. The obtained calibration curve and high AUC values confirmed that our nomogram model was reliable, and its predicting capability was robust. Therefore, using these models, we can predict pediatric patients' survival with BSGM more accurately and provide the basis for individualized treatment for these patients. Finally, the DCAs of the nomogram in 1-, 3-, and 5-year show that net benefits could be obtained in both the training and validation cohorts.

This study has some drawbacks. First, our study was based on the SEER database. This database lacks some vital information on tumor biological data, such as MGMT (O (6)-methylguanine-DNA methyltransferase),(Jovanović and Mitrović et al., 2019) promoter methylation, and IDH (isocitrate dehydrogenase) mutations,(de Quintana-Schmidt and Alvarez-Holzapfel et al., 2015) or H3 K27M,(Chi and Tarapore et al., 2019) which have substantial prognostic value in pediatric patients suffering from glioma. Secondly, it has no detailed description of the relationships with neurovascular structures and the precise location of the lesions in the brainstem. Thirdly, this is a retrospective study and cannot define causality. However, compared to other reported studies, it has the largest sample size, a strength.

\section{Conclusion}


Nomogram constructed with four variables in this study has a good performance. It is convenient, practical, and can help make the individual clinical decision and assess pediatric patients' risk with primary brainstem malignant glioma. This preoperatively predictive model was objective and accurate in predicting 1-, 3-, and 5-year CSS rates before treatment.

\section{Declarations}

Acknowledgments: I wish to thank Yujiao Wang and Didi Han for advice on research design.

Funding: No funding was received.

Conflicts of interest/Competing interests: The authors declare that no conflict of interest exists.

Availability of data and material: Clinicopathological data reported in this manuscript were collected from the SEER database. Copies of these data can be obtained free of charge from https://seer.cancer.gov.

Code availability: Not applicable.

Authors' contributions: Methodology, C.W.; software, C.W.; validation, L.C., L.J. and W.W.; formal analysis, C.W.; resources, C.W., L.C; writing-original draft preparation, C.W.; writing-review and editing, W.X., L.J.; supervision, W.X., L.J.; project administration, W.X. All authors have read and agreed to the published version of the manuscript.

Ethics approval: This study complies with the Declaration of Helsinki (World Medical Association Declaration of Helsinki). This study did not require ethics approval, as SEER database is publicly available and extraction of data from SEER were identified as nonhuman study.

Consent to participate: Not applicable as the SEER database was analyzed retrospectively.

Consent for publication: All the authors were agreed for publication freely.

\section{References}

Albright, A. L. and R. A. Price, et al. (1983). "Brain stem gliomas of children. A clinicopathological study." Cancer52(12): 2313-9.

Balachandran, V. P. and M. Gonen, et al. (2015). "Nomograms in oncology: more than meets the eye." The Lancet. Oncology.16(4): e173-80.

Bernier-Chastagner, V. and J. Grill, et al. (2005). "Topotecan as a radiosensitizer in the treatment of children with malignant diffuse brainstem gliomas: results of a French Society of Paediatric Oncology Phase II Study." Cancer104(12): 2792-7. 
Bjerke, L. and A. Mackay, et al. (2013). "Histone H3.3. mutations drive pediatric glioblastoma through upregulation of MYCN." Cancer discovery3(5): 512-9.

Chan, K. M. and D. Fang, et al. (2013). "The histone H3.3K27M mutation in pediatric glioma reprograms H3K27 methylation and gene expression." Genes \& development27(9): 985-90.

Chi, A. S. and R. S. Tarapore, et al. (2019). "Pediatric and adult H3 K27M-mutant diffuse midline glioma treated with the selective DRD2 antagonist ONC201." Journal of neuro-oncology 145(1): 97-105.

de Quintana-Schmidt, C. and M. J. Alvarez-Holzapfel, et al. (2015). "[Isocitrate dehydrogenase type I mutation as a prognostic factor in glioblastoma and a literature review]." Neurocirugia (Asturias, Spain).26(6): 276-83.

Dolecek, T. A. and J. M. Propp, et al. (2012). "CBTRUS statistical report: primary brain and central nervous system tumors diagnosed in the United States in 2005-2009." Neuro-oncology: v1-49.

Doyle, J. and A. M. Khalafallah, et al. (2019). "Association between extent of resection on survival in adult brainstem high-grade glioma patients." Journal of neuro-oncology.145(3): 479-486.

Esteve-Codina, A. and F. Alameda, et al. (2020). "RNA-Sequencing and immunohistochemistry reveal ZFN7 as a stronger marker of survival than molecular subtypes in G-CIMP-negative glioblastoma." Clinical cancer research : an official journal of the American Association for Cancer Research.

Fisher, P. G. and S. N. Breiter, et al. (2000). "A clinicopathologic reappraisal of brain stem tumor classification. Identification of pilocystic astrocytoma and fibrillary astrocytoma as distinct entities." Cancer89(7): 1569-76.

Freeman, C. R. and J. P. Farmer (1998). "Pediatric brain stem gliomas: a review." International journal of radiation oncology, biology, physics40(2): 265-71.

Fuchs, I. and W. Kreil, et al. (2002). "Gamma Knife radiosurgery of brainstem gliomas." Acta neurochirurgica. Supplement84: 85-90.

Grimes, D. A. (2008). "The nomogram epidemic: resurgence of a medical relic." Annals of internal medicine149(4): 273-5.

Guillamo, J. S. and A. Monjour, et al. (2001). "Brainstem gliomas in adults: prognostic factors and classification." Brain : a journal of neurology.124: 2528-39.

Hargrave, D. and U. Bartels, et al. (2006). "Diffuse brainstem glioma in children: critical review of clinical trials." The Lancet. Oncology.7(3): 241-8.

Hargrave, D. and U. Bartels, et al. (2006). "Diffuse brainstem glioma in children: critical review of clinical trials." The Lancet. Oncology.7(3): 241-8. 
Jallo, G. I. and A. Biser-Rohrbaugh, et al. (2004). "Brainstem gliomas." Child's nervous system : ChNS: official journal of the International Society for Pediatric Neurosurgery.20(3): 143-53.

Jiang, L. and T. Lin, et al. (2020). "A novel nomogram to predict the overall survival in esthesinoeroblastoma." BMC cancer20(1): 993.

Jovanović, N. and T. Mitrović, et al. (2019). "MGMTThe Impact of Promoter Methylation and Temozolomide Treatment in Serbian Patients with Primary Glioblastoma." Medicina (Kaunas, Lithuania).55(2).

Khalid, S. I. and R. Kelly, et al. (2019). "Pediatric Brainstem Gliomas: A Retrospective Study of 180 Patients from the SEER Database." Pediatric neurosurgery54(3): 151-164.

Lesniak, M. S. and J. M. Klem, et al. (2003). "Surgical outcome following resection of contrast-enhanced pediatric brainstem gliomas." Pediatric neurosurgery_39(6): 314-22.

Li, H. and Y. He, et al. (2020). "The Nomogram Model Predicting Overall Survival and Guiding Clinical Decision in Patients With Glioblastoma Based on the SEER Database." Frontiers in oncology.10: 1051.

Louis, D. N. and A. Perry, et al. (2016). "The 2016 World Health Organization classification of tumors of the central nervous system: a summary." Acta neuropathologica131(6): 803-820.

Marcus, K. J. and S. C. Dutton, et al. (2003). "A phase I trial of etanidazole and hyperfractionated radiotherapy in children with diffuse brainstem glioma." International journal of radiation oncology, biology, physics55(5): 1182-5.

Maxwell, R. and A. S. Luksik, et al. (2018). "Population-based Study Determining Predictors of CancerSpecific Mortality and Survival in Pediatric High-grade Brainstem Glioma." World neurosurgery.119: e1006e1015.

Moharamzad, Y. and M. Sanei Taheri, et al. (2018). "Brainstem glioma: Prediction of histopathologic grade based on conventional MR imaging." The neuroradiology_journal31(1): 10-17.

Mukherjee, D. and V. Antar, et al. (2020). "High-resolution diffusion tensor magnetic resonance imaging of the brainstem safe entry zones." Neurosurgical review43(1): 153-167.

Ostrom, Q. T. and H. Gittleman, et al. (2018). "CBTRUS statistical report: primary brain and other central nervous system tumors diagnosed in the United States in 2011-2015." Neuro-oncology_20(suppl_4): iv1iv86.

Pincus, D. W. and E. O. Richter, et al. (2006). "Brainstem stereotactic biopsy sampling in children." Journal of neurosurgery $104:$ 108-14. 
Qian, X. and H. Jia, et al. (2020). "Risk factors and prediction of second primary cancer in primary female non-metastatic breast cancer survivors." Aging12(19): 19628-19640.

Rengachary, S. and R. A. Rauf (2012). Principles of neurological surgery 3rd edition, Saunders.

Reyes-Botero, G. and K. Mokhtari, et al. (2012). "Adult brainstem gliomas." The oncologist17(3): 388-97.

Sanghavi, S. N. and M. N. Needle, et al. (2003). "A phase I study of topotecan as a radiosensitizer for brainstem glioma of childhood: first report of the Children's Cancer Group-0952." Neuro-oncology5(1): 813.

Schwartzentruber, J. and A. Korshunov, et al. (2012). "Driver mutations in histone H3.3 and chromatin remodelling genes in paediatric glioblastoma." Nature482(7384): 226-31.

Scott, R. M. (2004). "Surgical outcome following resection of contrast-enhanced pediatric brainstem gliomas." Pediatric neurosurgery.40(2): 99; author reply 100.

Stark, A. M. and M. J. Fritsch, et al. (2005). "Management of tectal glioma in childhood." Pediatric neurology33(1): 33-8.

Sun, T. and W. Wan, et al. (2013). "Clinical outcomes and natural history of pediatric brainstem tumors: with 33 cases follow-ups." Neurosurgical review36(2): 311-9; discussion 319-20.

Sun, T. and Y. Xu, et al. (2020). "Surgical treatment and prognosis of focal brainstem gliomas in children: A 7 year single center experience." Medicine99(36): e22029.

Theeler, B. J. and B. Ellezam, et al. (2015). "Adult brainstem gliomas: Correlation of clinical and molecular features." Journal of the neurological sciences353: 92-7.

Wang, Y. and J. Li, et al. (2013). "Prognostic nomogram for intrahepatic cholangiocarcinoma after partial hepatectomy." Journal of clinical oncology: official journal of the American Society of Clinical Oncology31(9): 1188-95.

Wong, J. F. and E. J. Brown, et al. (2019). "Fostering open collaboration in drug development for paediatric brain tumours." Biochemical Society transactions47(5): 1471-1479.

Yang, Z. and Y. Bai, et al. (2020). "Development and validation of a prognostic nomogram for predicting cancer-specific survival after radical cystectomy in patients with bladder cancer:A population-based study." Cancer medicine.

Zhu, J. and X. Zhu, et al. (2016). "Parity and thyroid cancer risk: a meta-analysis of epidemiological studies." Cancer medicine5(4): 739-52.

\section{Tables}




\begin{tabular}{cccccc}
\hline Characteristics Categories & Total (\%) & Training (\%) Validation (\%)P Value \\
\hline Age & 0y-9y & $819(70.60)$ & $568(69.95)$ & $251(72.13)$ & 0.456 \\
& 10y-18y & $341(29.40)$ & $244(30.05)$ & $97(27.87)$ & \\
\hline Years & Before 2003 & $310(26.72)$ & $232(28.57)$ & $78(22.41)$ & 0.030 \\
& After 2003 & $850(73.28)$ & $580(71.43)$ & $270(77.59)$ & \\
\hline \multirow{2}{*}{ Sex } & Male & $562(48.45)$ & $397(48.89)$ & $165(47.41)$ & 0.644 \\
& Female & $598(51.55)$ & $415(51.11)$ & $183(52.59)$ & \\
\hline Race & White & $864(74.48)$ & $602(74.14)$ & $262(75.29)$ & 0.905 \\
& Black & $187(16.12)$ & $132(16.26)$ & $55(15.80)$ & \\
& Other & $109(9.40)$ & $78(9.61)$ & $31(8.91)$ & \\
\hline Histological & Low-grade & $70(6.03)$ & $44(5.42)$ & $26(7.47)$ & 0.236 \\
grade & High-grade & $84(7.24)$ & $55(6.77)$ & $29(8.33)$ & \\
& Mixed & $1006(86.72)$ & $713(87.81)$ & $293(84.20)$ & \\
\hline Size(mm) & $\leq 25$ & $166(14.31)$ & $121(14.90)$ & $45(12.93)$ & 0.540 \\
& $\mathbb{2} 5$ & $545(46.98)$ & $374(46.06)$ & $171(49.14)$ & \\
& Other & $449(38.71)$ & $317(39.04)$ & $132(37.93)$ & \\
\hline Laterality & Right & $19(1.64)$ & $13(1.60)$ & $6(1.72)$ & 0.699 \\
& Left & $18(1.55)$ & $11(1.35)$ & $7(2.01)$ & \\
\hline Months & Other & $1123(96.81)$ & $788(97.04)$ & $335(96.26)$ & \\
\hline & & $12(7-27.25)$ & $12(7-29.5)$ & $12(7-26)$ & 0.315 \\
\hline
\end{tabular}

Table 1 Demographics and clinicopathological characteristics of Pediatric brainstem malignant gliomas.

\section{Figures}



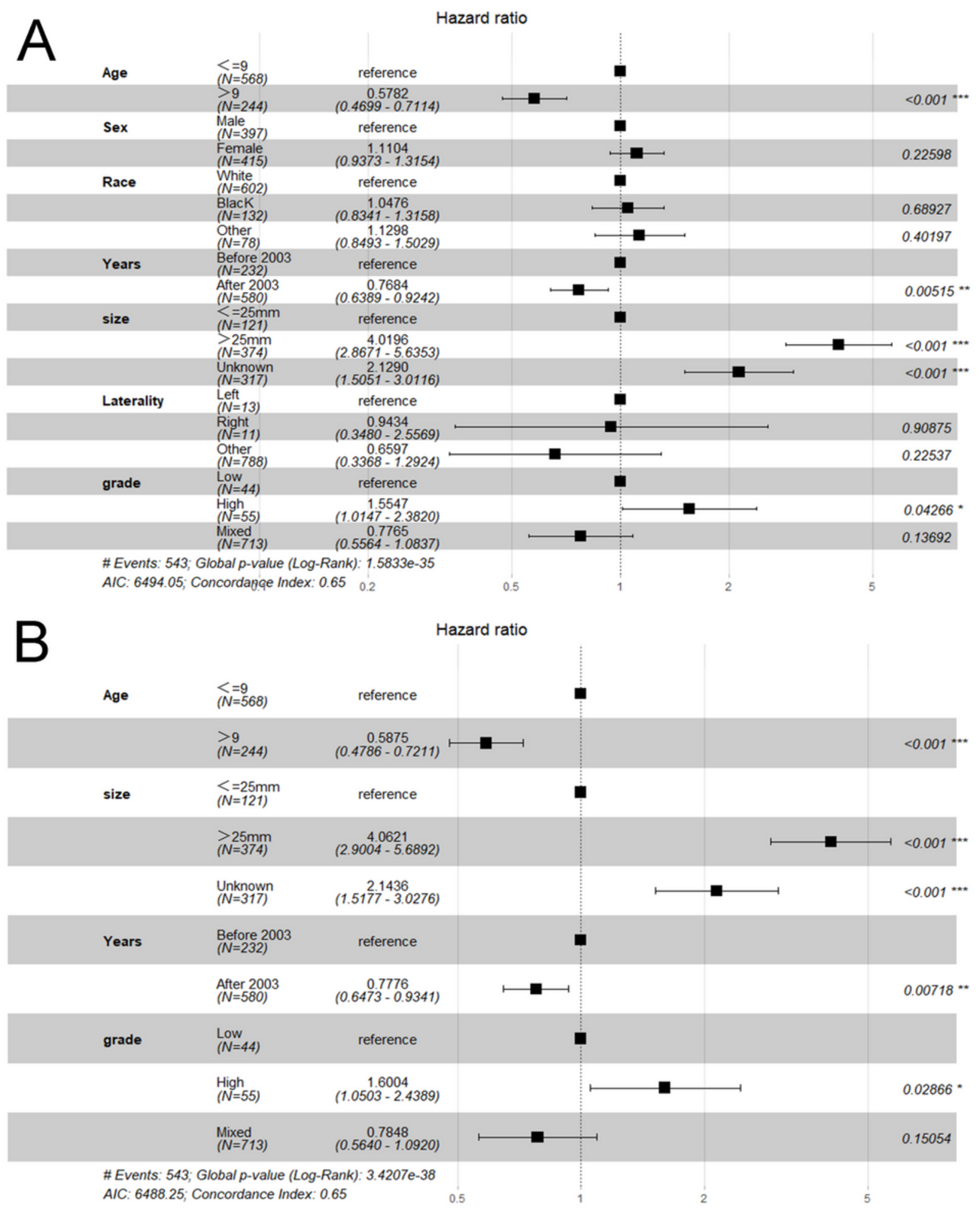

\section{Figure 1}

Multivariate Cox analysis (A) and backward stepwise regression analysis (B) of cancer-specific survival factors! 


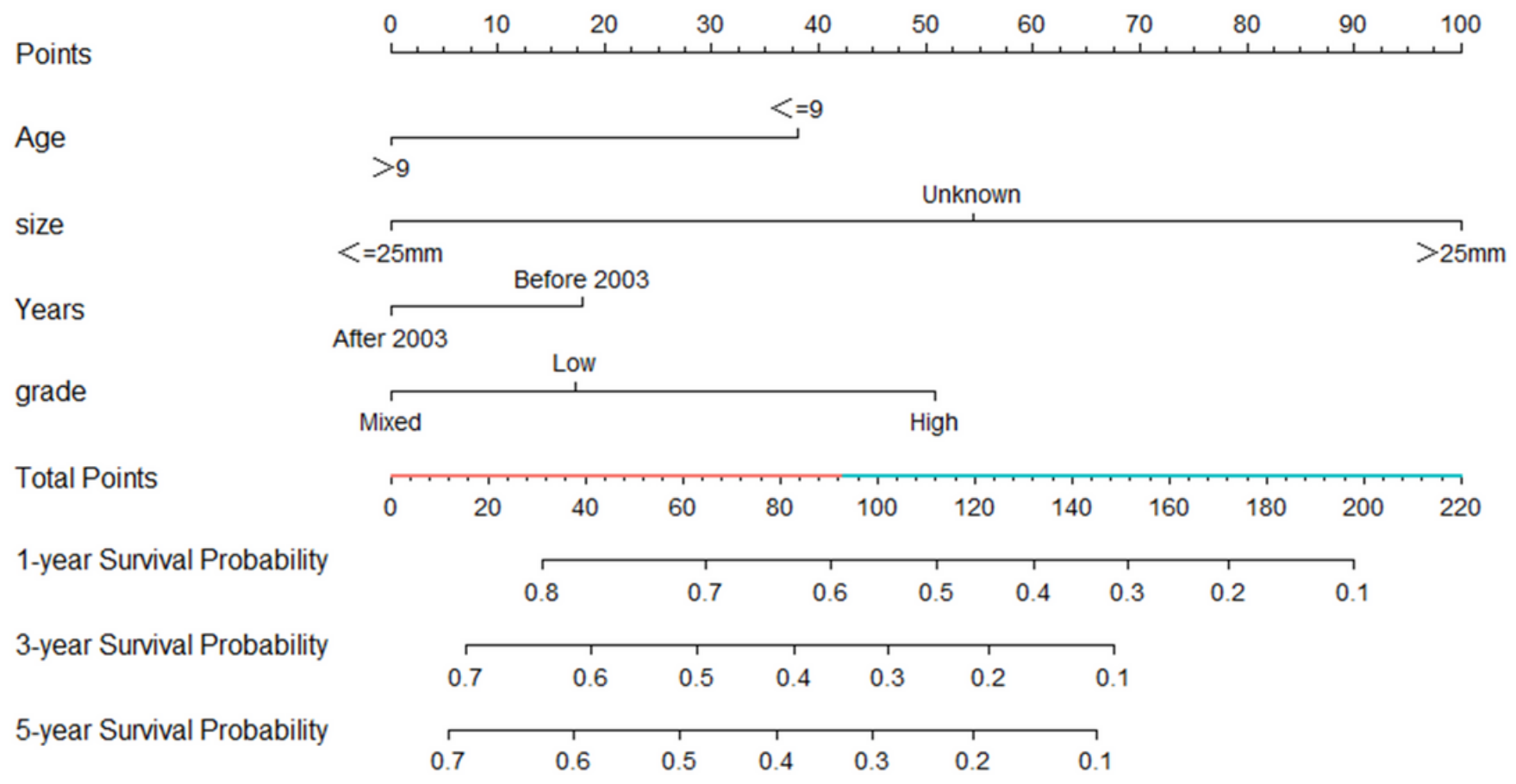

Figure 2

A developed nomogram for prognostic prediction of pediatric brainstem malignant gliomas cancerspecific survival rates at 1-, 3-, and 5-year. Patients were divided into two risk classifications: low risk

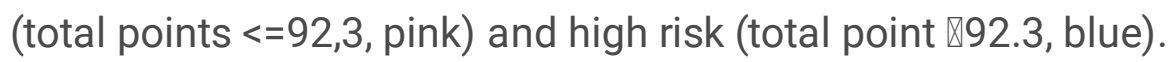


A

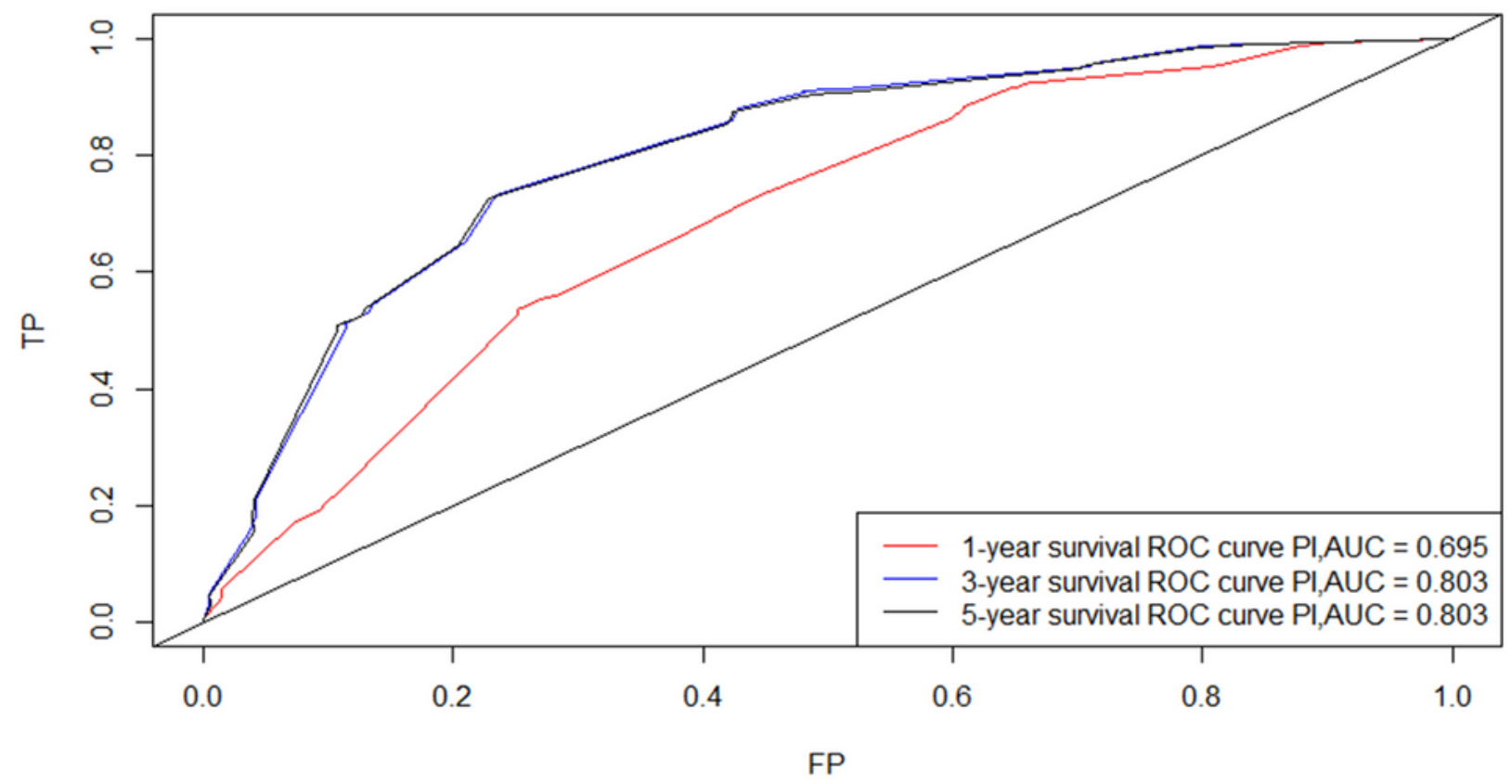

\section{B}

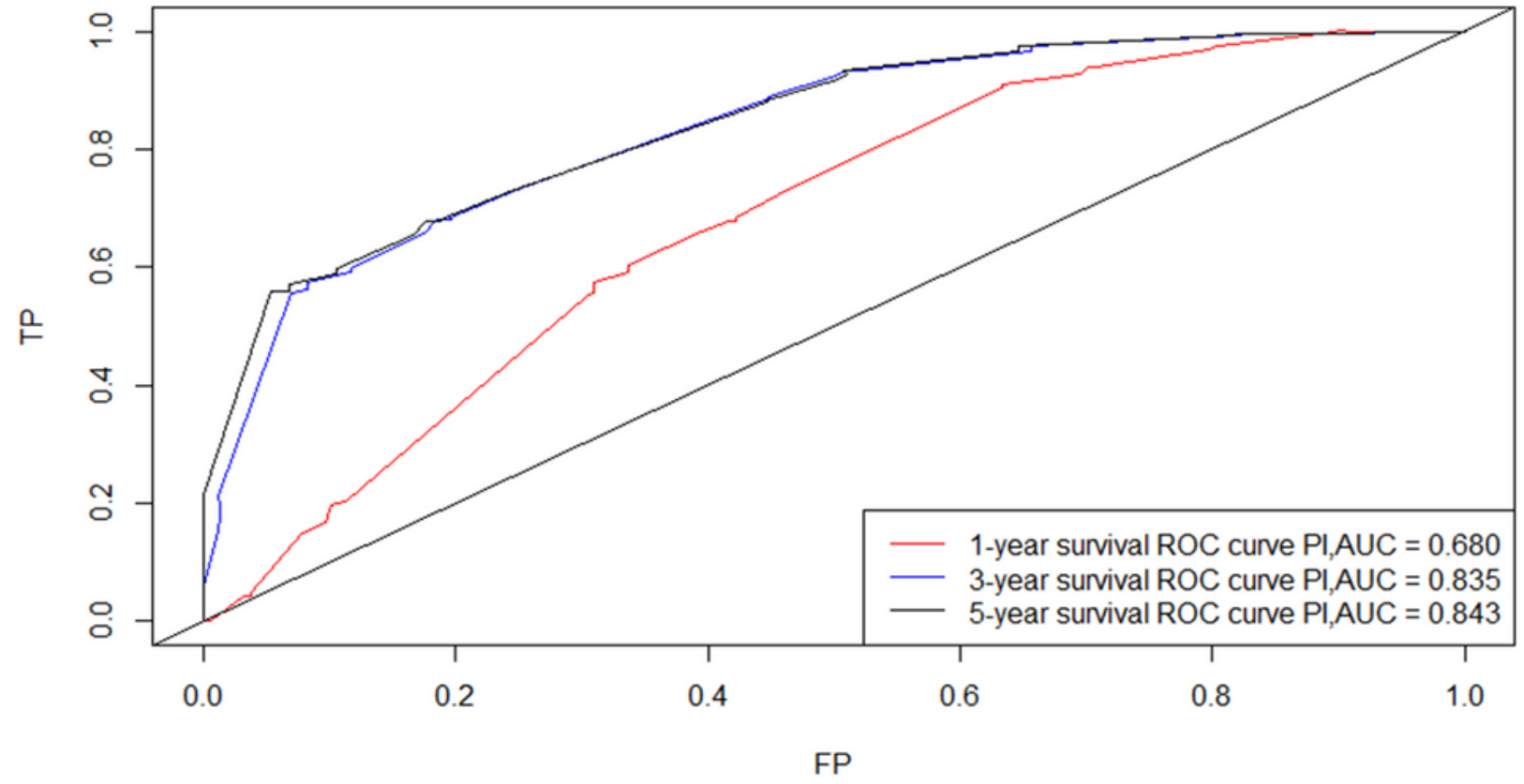

Figure 3

ROCs curve for nomograms. AUCs of the nomograms to predict cancer-specific survival at 1-, 3-, and 5year using training dataset $(A)$ and validation dataset $(B)$. 

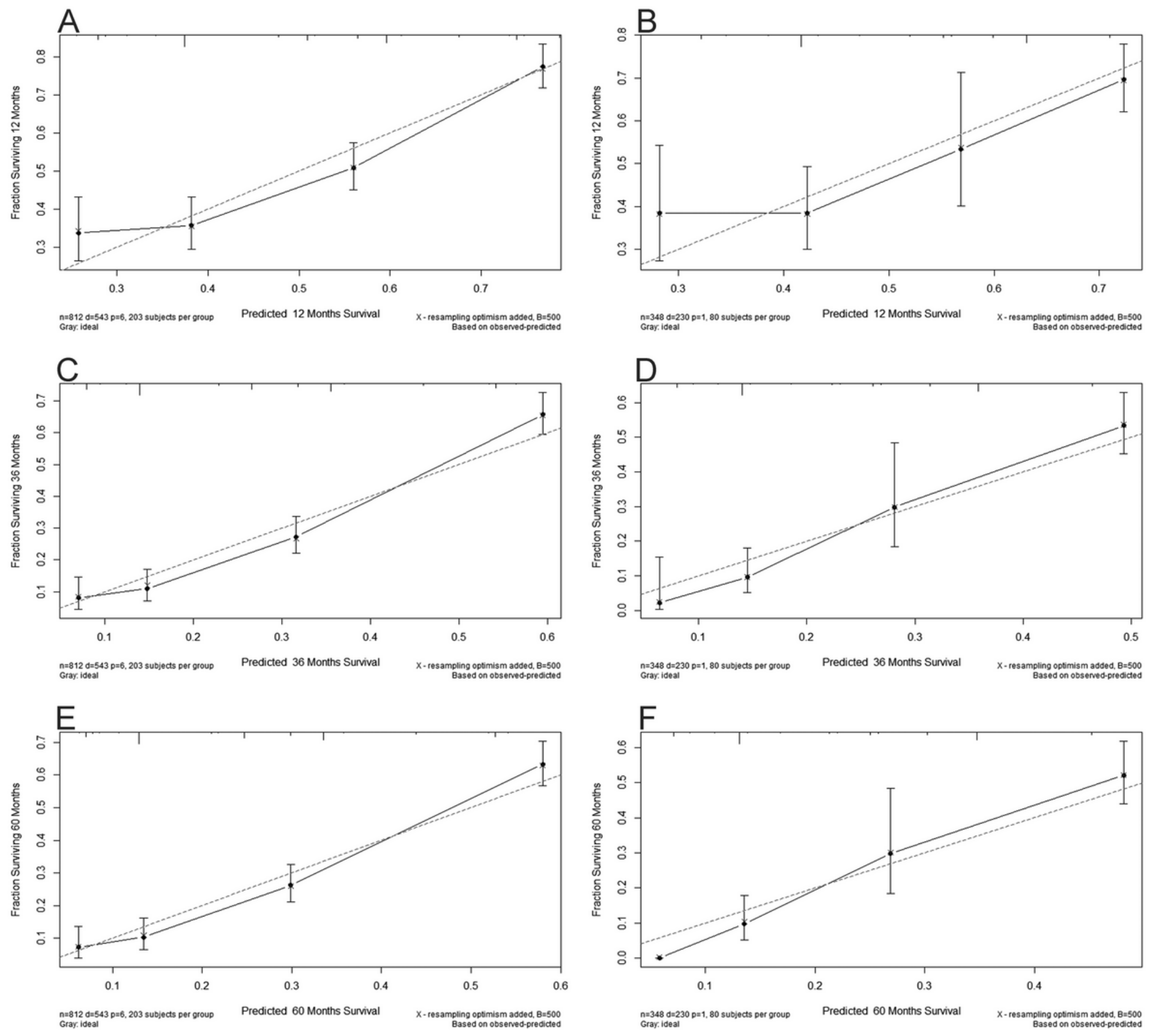

Figure 4

The calibration curves in training $(A, C, E)$ and validation $(B, D, F)$ cohorts show the nomogram with excellent predicting ability in 1-, 3-, and 5-year cancer-specific survival $\square$ 

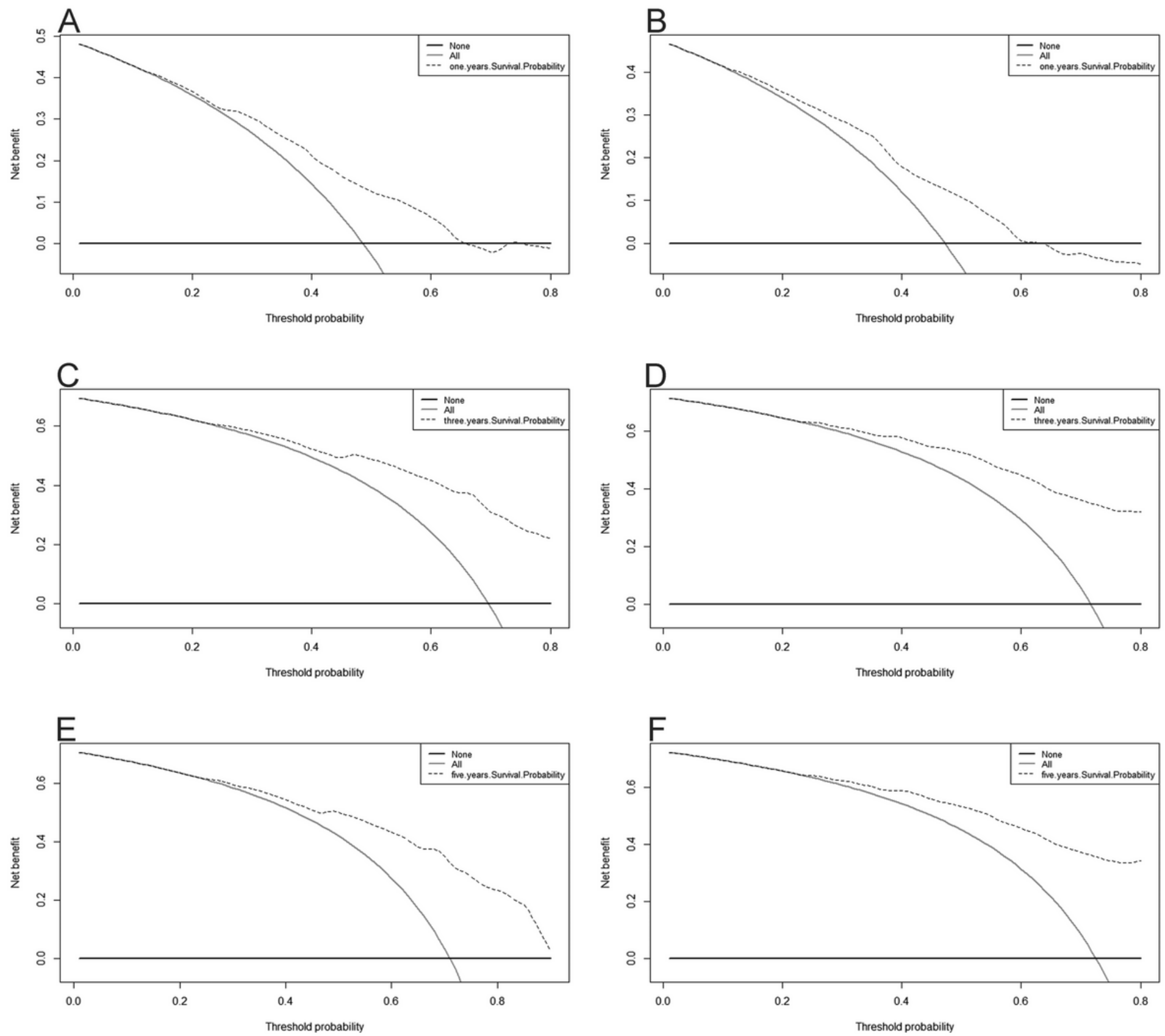

Figure 5

DCAs of a nomogram for cancer-specific survival in 1-, 3-, and 5-year show that both the training and validation cohorts could obtain net benefits. A, C, and E came from the training group, and the rest came from the validation group. 

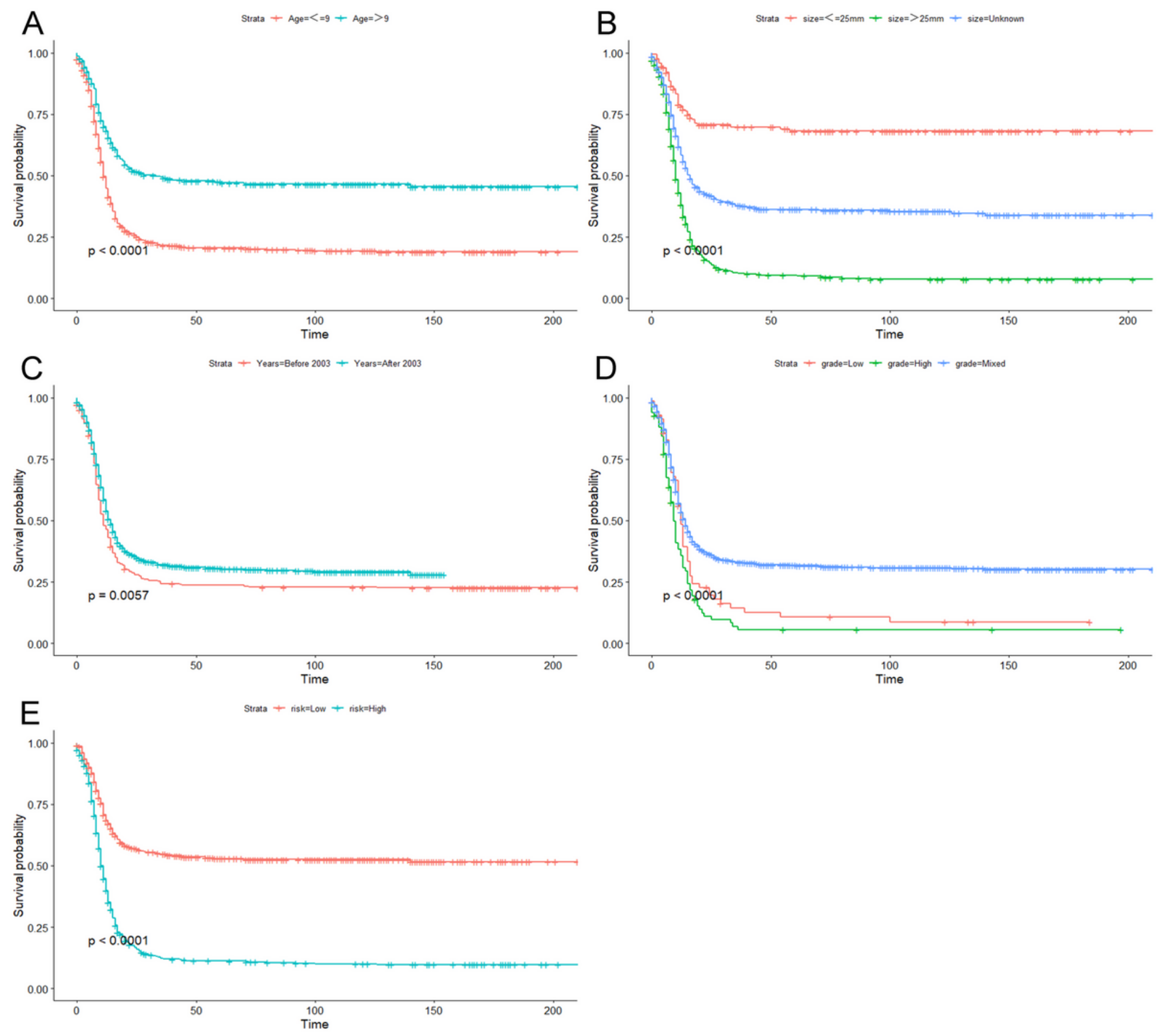

Figure 6

Kaplan-Meier cancer-specific survival curves of pediatric BSMGs stratified by age at diagnosis (A), tumor size (B), years at diagnosis(C), histological grade (D), and risk group stratification (E). 

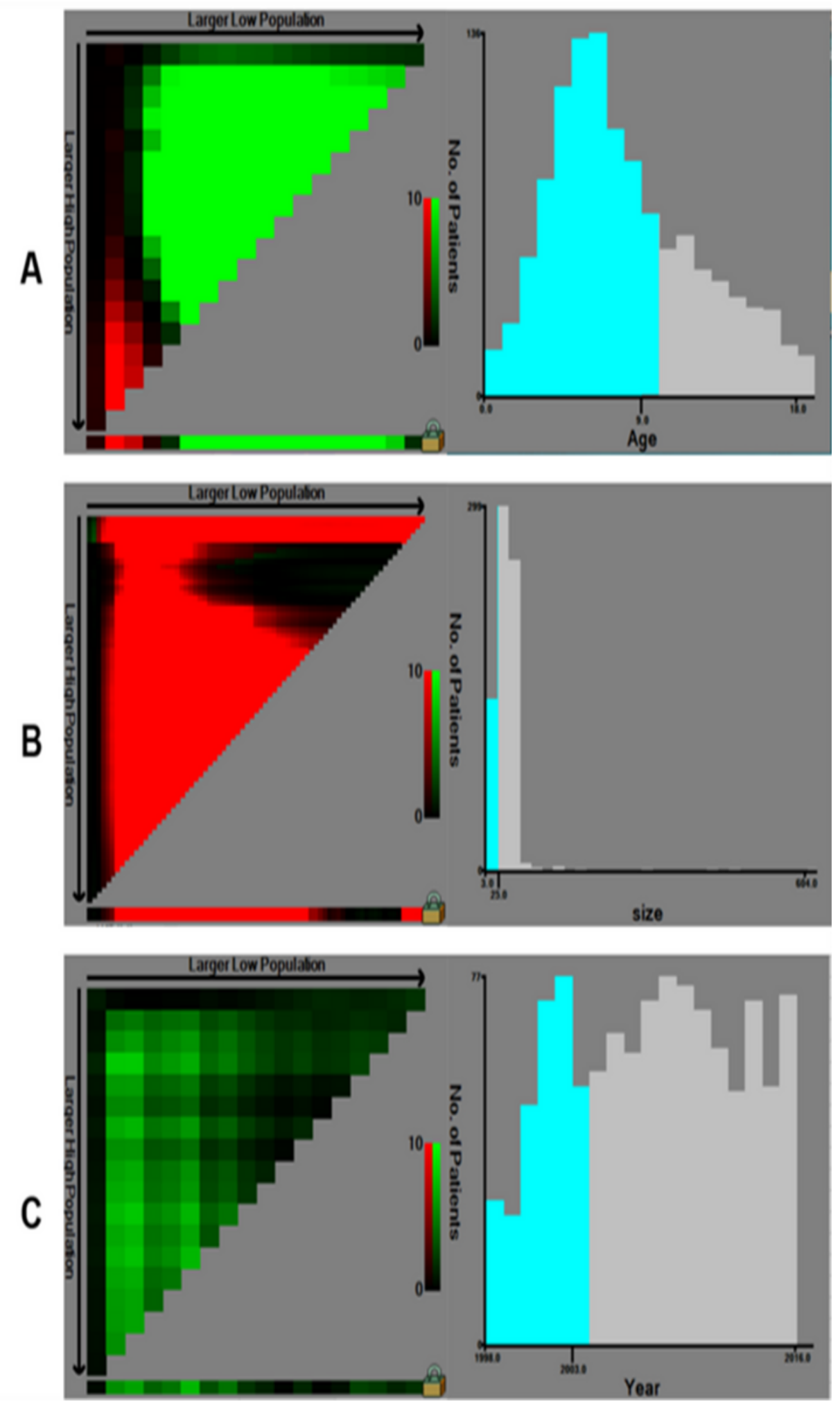

Figure 7

Age at diagnosis (A), tumor size (B) years at diagnosis (C) stratification using the X-tile software. 